\title{
THORACIC CHORDOMA
}

\section{Rita Delgado', Antonio de Pádua Furquim Bonatelli², João Norberto Stávale}

Chordomas are slow-growing tumors with aggressive local behavior that probably originate from embryonic remnants of the notochord. This is a midline structure along the neural tube that presents involution and fragmentation as ossification of the axial skeleton occurs, in such way that in the second month of intrauterine life, it is restricted to the intervertebral discs, where the nucleus pulposus will appear. Other remnants of the notochord originating outside the discs and within the bones can be found in any topography long the vertebral column but they are preferably found in the sacrococcygeal region ( 50 to $60 \%$ ) or in the sphenobasilar region ( 25 to $40 \%$ of the cases). In the remaining portion of the vertebral column (15\%) the main localization is the cervix, especially the high cervical spine, followed by the lumbar spine'. Chordomas of the thoracic spine are rare and usually present as extradural bone tumors that lead to complaints of pain by instability and intercostal radiculalgia or neurological changes in the lower limbs due to spinal cord compression ${ }^{2-5}$.

We report the case of a thoracic chordoma that presented as a pathological fracture and received a clinical and initial radiological diagnosis of vertebral metastasis.

\section{CASE}

A 68 years old woman was admitted to the emergency room at Hospital Sâo Paulo with a history of pain in the upper abdomen for the previous three months. She reported the pain as ranging from moderate to intense and in a strip, irradiating to the flanks. In the previous ten days she started presenting weakness of the lower limbs, rapidly evolving to paralysis and urinary incontinence. Around admission, the patient presented flaccid paraplegia, global anesthesia below T4 and atonia of the anal sphincter (Frankel A).

Radiographies of the thoracic spine evidenced fracture with jamming of the T7 body and lytic lesion of the pedicle and transverse process at this level. Magnetic resonance image (MRI) revealed an isointense tumor in the sagittal T1-weighted sequences, of lytic aspect, determining fracture of the vertebral body of T7, with compression of the spinal cord at this level. The axial cuts showed the typical "curtain sign" of epidural involvement and infiltration of the pedicle, costovertebral joint and transverse process to the right. The T2-weighted sequences showed signal hyperintensity at the lesion level and gadolinium injection slightly highlighted it. The radiological aspects of pathological

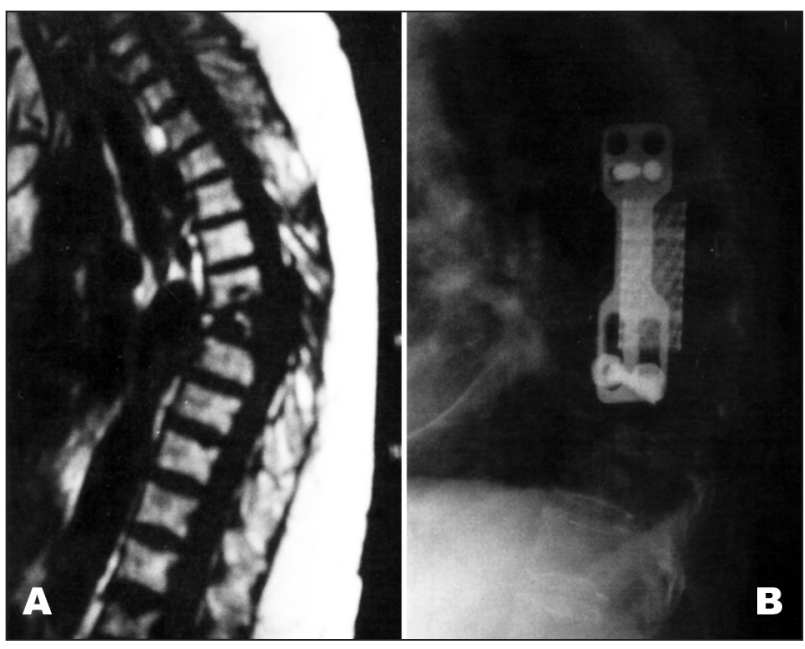

Fig $1(A, B)$. Postoperative anteroposterior and lateral radiographies showing cage placement and lateral locking plate for T6-T8 arthrodesis.

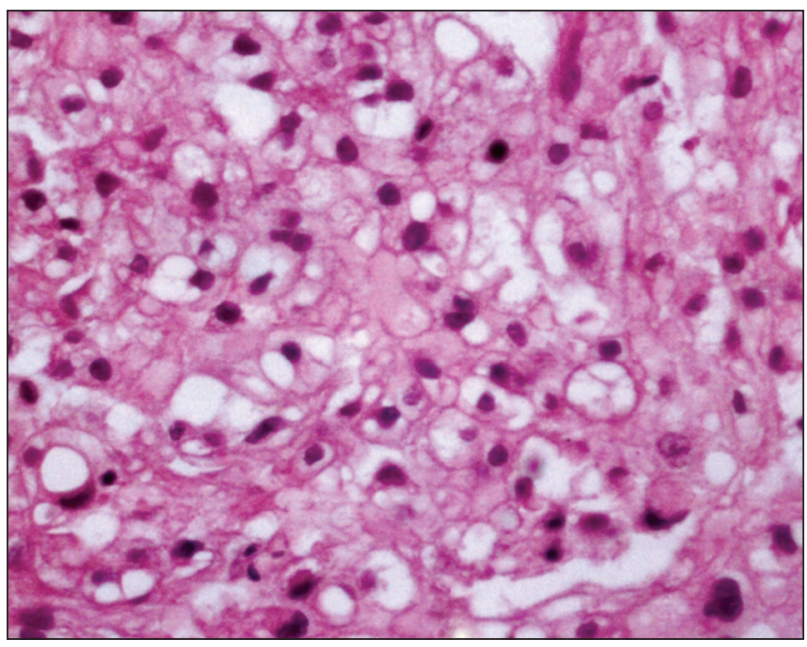

Fig 2. Histopathology. The physaliphora cells are oval, have a central nucleus and vacuolated cytoplasm and are soaked in myxomatous and eosinophilic stroma.

\section{CORDOMA TORÁCICO}

Hospital São Paulo - Escola Paulista de Medicina, São Paulo SP, Brazil (UNIFESP): 'Neurosurgeon, Master in Sciences, Neurosurgery, UNIFESP; ${ }^{2}$ Associate Professor, Neurosurgery, UNIFESP; ${ }^{3}$ Associate Professor, Pathological Anatomy, UNIFESP.

Received 9 October 2007, received in final form 28 March 2008. Accepted 17 April 2008.

Dra. Rita Delgado - Rua Borges Lagoa 1080 / 307 - 04038-002 São Paulo SP - Brasil. E-mail: ritadelg@yahoo.com.br 
fracture and lytic bone lesion with epidural involvement in the thoracic spine were initially interpreted as metastasis.

The patient was submitted to posterior access by right costotransversectomy to decompress the fracture and remove material for pathological study. The tumor was firm, brown and bled little upon handling. The patient tolerated the procedure well but the neurological picture remained unchanged. She was discharged from the hospital one week later and was set for follow-up. The pathological examination proved the mass to be a chordoma.

The family of the patient contacted the team 8 months later to report that the patient was improving slowly, walking with help and that she was again feeling pain. She was hospitalized again and presented an amazing neurological recovery, with paraparesis (degrees 3 and 4 of muscle strength) with spasticity and hyperreflexia, sensitivity disorders or sphincter disorders (Frankel D). New radiological investigation revealed recurrence of the tumor at the same level with more mass than before, when initially diagnosed, and paravertebral extension lobulated to the right.

The patient was submitted to a transpleural thoracotomy approach for T7 corpectomy and T6-T8 anterior arthrodesis. The tumor had a lobulated, whitish and externally consistent aspect and was involved by a capsule that delimited it from the adjacent structures. It was clearly continuing from the remaining bone tissue of the vertebral body of T7. The tumor did not infiltrate the intervertebral discs. The tumor mass was excised in pieces, corpectomy of T7 was performed and the intervertebral discs above and below were removed for arthrodesis. Arthrodesis was done by interposition of the cage where the intervertebral faces below T6 and above T8 were filled with ceramic and the Zdeblick plate was fixed, with a satisfactory intraoperative radioscopic control (Fig 1A, 1B).

In the immediate postoperative period the patient evolved with good neurological recovery. One week after the surgery circulatory instability by infection was detected but the patient improved with antibiotic treatment. Eleven days after surgery the patient presented ischemia of the right hand due to lesion of the radial artery, catheterized for continuous blood pressure monitoring. The picture evolved to necrosis due to arterial insufficiency and amputation of the first, second and third fingers was necessary. Specific antibiotic therapy was maintained and the wound was debrided again twenty days later, but unfortunately the patient presented a new circulatory instability episode due to sepsis and died 74 days after admission.

\section{DISCUSSION}

In 1856 Luschka described the presence of small, transparent, gelatinous nodules in the prepontine region, projecting from the clivus in post mortem studies'. In the following year, it was Virchow who presented a histological description of these nodulations, suggesting a cartilagi- nous origin for them and naming them ecchordosis physaliphora ${ }^{2}$. Based on anatomical and embryological evidences, Friedrich von Müller suggested in 1858 that the origin of these lesions would be in notochord remnants ${ }^{3}$. Moritz Ribbert performed an experimental study in rabbits 40 years later to try to analyze this hypothesis ${ }^{4}$. He created disc herniations by puncturing the intervertebral discs and analyzed the herniated material after one year of progress. The histopathological studies showed the formation of a gelatinous tissue, very similar to that of the notochord, which corresponded to Virchow's previous description of ecchordosis physaliphora ${ }^{3}$. The microscopic aspect of the chordoma, similar to immature cartilage with oval cells, centralized nucleus and vacuolated cytoplasm (characteristic of physaliphora cells) suggests some connection between the two lesions, but if the tumor really originates from the notochord or from a previously benign lesion such as ecchordosis, it is pure speculation'.

The chordomas account for 3 to $4 \%$ of the tumors with malignant behavior found in the skeleton. They affect males more often than females in the ratio of $2: 1^{4}$. They may occur at any age but the incidence peak happens between the fifth and sixth decades of life. In 50 to $60 \%$ of the cases, they affect the sacrococcygeal region and in 25 to $40 \%$ of the cases, the sphenobasilar region; in this group the patients can be a little younger ${ }^{1}$. When they affect the cervical column they usually do so in the upper segments. The lumbar location is considered uncommon and the thoracic is considered rare. Sundaresan and Galicich found 2 chordomas located in the thoracic spine in a series of 54 patients with proved histopathological diagnosis. Volpe and Mozabraud published a series of 25 spinal chordomas and none were in the thoracic region. In the largest retrospective series done until now, Utne and Pugh (Mayo Clinic, 1954) found only 20 thoracic chordomas in 1271 diagnosed chordomas ${ }^{1-4}$.

The clinical manifestation of the thoracic chordomas is usually insidious and progressive, with signs of pain by spine instability, a strip of radiculalgia by compression of the thoracic roots or syndromes by compression of the spinal cord by the tumor. These pictures of myelopathy may worsen suddenly when a pathological fracture of the vertebra happens, with sudden reduction of the spinal canal. Sympathetic involvement in upper thoracic lesions was described in an 18-year old patient whose expression of a chordoma between $\mathrm{T} 1$ and $\mathrm{T} 2$ was a Claude-BernardHorner syndrome ${ }^{6}$. Recurrent dry cough was the clinical manifestation of a chordoma in a 14-year old patient, with a cystic form of the tumor growing from T3 and T4 which extended to the upper mediastine, posteriorly. Eight similar cases are reported in the literature ${ }^{7}$. 
From the radiological point of view, chordoma diagnosis should be considered in destructive lesions of the vertebral body associated with a mushroom-like or hourglass-like mass that extends to many vertebral levels and preserves the intervertebral discs. The extension may happen in an anterior, lateral or posterior direction, affecting the epidural space and giving the image the characteristic "curtain sign" in the axial cuts'. Calcifications can occur in 40 to $70 \%$ of the cases ${ }^{1,6}$. Studies by MRI demonstrate a usually isointense lesion in T1-weighted sequences and hyperintense in $\mathrm{T} 2$, slightly highlighted with gadolinium injection.

Jallo et al..$^{8}$ propose a radiological classification for chordomas according to the planes that affect the vertebral column: type I when the lesion is osseous extradural (most); type II is extraosseous extradural; type III is osseous intradural; and type IV is extraosseous intradural. The classification has a prognostic value since lesions of type II and IV (those that do not involve the bone) are easier to resect and more likely to be cured. Unfortunately these lesions represent the minority of the cases. They are exclusively epidural tumors that grow in a posterolateral situation, broadening the neuroforamen and mimicking a tumor of the nerve sheath ${ }^{3,9}$. The case reported here is classified as type I: osseous extradural lesion, with pathological fracture causing pain and compression of the spinal cord. Metastases, osteosarcomas, chondrosarcomas, plasmocytomas, osteomyelitis, schwannomas, neurofibromas, ependymomas, lymphomas and spinal meningiomas are the differential diagnosis of these lesions in the thoracic spine ${ }^{1,10}$.

Macroscopically, chordomas are tumors of whitish to brownish color, soft, multilobulated and delimited by a fibrous pseudocapsule, with a gelatinous nucleus where areas of hemorrhage and necrosis are found. Microscopically, they consist of physaliphora cells that are oval, have a central nucleus and vacuolated cytoplasm and are soaked in myxomatous and eosinophilic stroma (Fig 2). Chordomas may have a component of associated cartilaginous tissue and in this case they are classified as chondroid chordomas. It may be hard to distinguish these from chondrosarcomas. The differential diagnosis involves chondrosarcoma, metastatic carcinoma and myxopapillary ependymoma. Metastatic carcinomas and chordomas stain positively for epithelial markers. However, chordomas are, in addition, positive for S-100 protein. Chondro- sarcomas and myxopapillary ependymomas do not stain with epithelial markers. The lobulated growth pattern with fibrous septa is characteristic for chordomas. Chondrosarcomas tend to be lobulated, but they do not show fibrous septa. Another possible variant is the dedifferentiated chordomas that present areas of associated pleomorphic sarcomatous component. Truly malignant components, such as vacuolization of the nuclei and figures of mitosis are exceptional. Peters (1919), Lewis (1921) and Pototschin (1919) describe cases of malignant chordomas with metastases to the lymph nodes and liver, but these are extremely rare reports ${ }^{1-3}$.

A surgical approach with block-excision of the tumor is the ideal treatment for managing chordomas. As mentioned before, it is rarely possible to fully resect the tumor ${ }^{8}$. The approach by thoracotomy for the aggressive exeresis of the tumor and later stabilization of the vertebral column with a rigid fixation material turned out to be the surgical treatment of choice in cases where vertebral bodies are affected after previous biopsy is done to establish the diagnosis ${ }^{1,2,4-6,11}$. Since the tumor does not respond well to radiotherapy, survival depends on resection and can be of up to 5 years in $75 \%$ of the cases where a satisfactory macroscopic exeresis is done ${ }^{6}$.

\section{REFERENCES}

1. Smolders D, Wang X, Drevelengas A, Vanhoenacker F, De Schepper AM. Value of MRI in the diagnosis of non-clival, non-sacral chordoma. Skeletal Radiol 2003;32:343-350.

2. Bellotti C, Ettorre F, Oliveri G, Medina M, Barrale S. Chordome vertebral dorsal a expansion endothoracique: traitement chirurgical d'un cas. Neurochirurgie 1986;32:86-89.

3. Rengachary SS, Grotte DA, Swanson PE. Extradural ecchordosis physaliphora of the throracic spine:case report. Neurosurgery 1997;41: 1198-1021

4. Schwarz SS, Fisher WS, Pulliam MW, Weinstein ZR. Thoracic chordoma in a patient with paraparesis and ivory vertebral body. Neurosurgery 1985;16:100-102.

5. Walsh TM, Mayer PJ. Chordoma of the thoracic spine presenting as a second primary malignant lesion. Spíne 1992;17:1524-1528.

6. Winants D, Bertal A, Hennequin L, Fays J, Bernadac P. Imagerie des chordomes cervicaux et thoraciques: à propos de 2 observations. J Radiologie 1992;73:169-174.

7. Cotler HB, Cotler JM, Cohn HE, Israel HI, Gartland JJ. Intrathoracic chordoma presenting as a posterior superior mediastinal tumor. Spine 1983;8:781-786.

8. Jallo J, Nathan D, Bierbauer K, Farber E. Chordoma: a case report. Surg Neurol 1997;48:46-48.

9. Holden A, Khangure M, Robins P. Thoracic chordoma mimicking a neurogenic tumor. Australasian Radiology 1998;42:385-387.

10. Samuel KC, Singh KN, Garg RK. Malignant chordoma of cervicothoracic region-a case report. Indian J Path Bact 1969;12:122-125.

11. Murphy JM, Wallis F, Toland J, Toner M, Wilson GE. CT and MRI appearances of a thoracic chordoma. Eur Radiol 1998;8:1677-1679. 